\title{
Differences in breast cancer characteristics and outcomes between Caucasian and Chinese women in the US
}

\author{
Dan-Na Chen ${ }^{1, *}$, Chuan-Gui Song ${ }^{1, *}$, Qian-Wen Ouyang ${ }^{2,3, *}$, Yi-Zhou Jiang ${ }^{4, *}$, Fu-Gui \\ $\mathrm{Ye}^{1}$, Fang-Jing $\mathrm{Ma}^{4}$, Rong-Cheng $\mathrm{Luo}^{2}$, Zhi-Ming Shao ${ }^{4}$ \\ ${ }^{1}$ Department of Breast Surgery, Affiliated Union Hospital, Fujian Medical University, Fuzhou, China \\ ${ }^{2}$ Cancer Center, Traditional Chinese Medicine-Integrated Hospital, Southern Medical University, Guangzhou, China \\ ${ }^{3}$ Department of Breast Surgery, The Third Hospital of Nanchang, Nanchang, China \\ ${ }^{4}$ Department of Breast Surgery, Key Laboratory of Breast Cancer, Fudan University Shanghai Cancer Center, Shanghai \\ Medical College, Fudan University, Shanghai, China \\ * These authors have contributed equally to this work. \\ Correspondence to: Yi-Zhou Jiang, email: yizhoujiang@fudan.edu.cn \\ Chuan-Gui Song, email: songchuangui@yahoo.com \\ Rong-Cheng Luo, email: Ivorc01@163.com
}

Keywords: breast cancer, clinicopathological characteristics, prognosis, Chinese, Caucasian

Received: February 06, $2015 \quad$ Accepted: February 28, $2015 \quad$ Published: March 26, 2015

This is an open-access article distributed under the terms of the Creative Commons Attribution License, which permits unrestricted use, distribution, and reproduction in any medium, provided the original author and source are credited.

ABSTRACT

Chinese breast cancer patients living in the United States (US) can experience different disease patterns than Caucasians, which might allow for predicting the future epidemiology of breast cancer in China. We aimed to compare the clinicopathologic characteristics and outcomes of Caucasian and Chinese female breast cancer patients residing in the US. The study cohort consisted of 3868 Chinese and 208621 Caucasian women (diagnosed from 1990 to 2009) in the US Surveillance, Epidemiology, and End Results (SEER) database. Compared with the Caucasian patients, the US-residing Chinese patients had a younger age at diagnosis and a higher family income, remained married longer, and more frequently lived in metropolitan areas. Other tumor characteristics were similarly distributed between the two races. Compared with the Caucasians, the Chinese patients had a significantly improved overall survival (OS) but similar breast cancer-specific survival (BCSS). Our analysis suggested that US-residing Chinese patients had significant differences in age, family income, marital status and area of residence, compared with their Caucasian counterparts. No significant disparities were noted in BCSS between the two races, whereas the Chinese patients had a significantly better OS. These findings warrant further investigation and should be considered in the screening and treatment of breast cancer.

\section{INTRODUCTION}

It has been well established that racial disparities exist in the incidence, mortality, and survival of patients with breast cancer. In China, breast cancer has a lower incidence and mortality compared with developed Western countries [1]. However, Chinese patients appear to have worse survival rates than Western women [2]. These discrepancies might be due to differences between the genetic backgrounds of Asians and Caucasians, as well as social-environmental factors, such as economic status, culture, lifestyle, and dietary habits. In addition, the incidence of breast cancer in China has been increasing rapidly [2,3], and some researchers have speculated that the incidence curve will be similar to that in Western countries in the future [1], possibly due to an increase in economic status and alterations in lifestyles.

When Chinese people immigrate to high-income Western countries such as the United States (US), they frequently change their lifestyles to adapt to Western culture [4]. Once immigrants have left their native countries of origin, they might expose themselves 
Table 1: Clinicopathologic characteristics of Caucasian and Chinese female breast cancer patients residing in the US from the SEER database.

\begin{tabular}{|c|c|c|c|}
\hline \multirow{2}{*}{ Characteristics } & \multicolumn{2}{|c|}{ No. $(\%)$ of Patients } & \multirow{2}{*}{$\mathrm{P}^{\mathrm{a}}$} \\
\hline & Chinese & Caucasian & \\
\hline Total & 3,868 & 208,621 & \\
\hline Age (mean) (years) & 53.60 & 57.30 & \\
\hline Age at Diagnosis (years) & & & $<0.01$ \\
\hline $20-39$ & $407(10.52)$ & $14,680(7.04)$ & \\
\hline $40-59$ & $2,305(59.59)$ & $103,119(49.43)$ & \\
\hline $60-79$ & $1,156(29.89)$ & $90,882(43.56)$ & \\
\hline Year of Diagnosis & & & $<0.01$ \\
\hline $1990-1999$ & $1,011(26.14)$ & $48,319(23.16)$ & \\
\hline $2000-2009$ & $2,857(73.86)$ & $160,302(76.84)$ & \\
\hline Marital Status & & & $<0.01$ \\
\hline Married & $2,876(74.35)$ & $135,306(64.86)$ & \\
\hline Not married ${ }^{\mathrm{b}}$ & $992(25.65)$ & $73,315(35.14)$ & \\
\hline Family Income (mean) (\$) & $83,231.58$ & $73,049.72$ & \\
\hline Family Income (\$) & & & $<0.01$ \\
\hline$<61,790$ & $106(2.74)$ & $52,872(25.34)$ & \\
\hline $61,790-69,700$ & $962(24.94)$ & $52,132(24.99)$ & \\
\hline $69,700-85,140$ & $631(16.31)$ & $49,982(23.96)$ & \\
\hline$\geq 85,140$ & $2,169(56.08)$ & $52,638(25.23)$ & \\
\hline County Type & & & $<0.01$ \\
\hline Metropolitan & $3,676(95.04)$ & $186,052(89.18)$ & \\
\hline Nonmetropolitan & $192(4.96)$ & $22,571(10.82)$ & \\
\hline Laterality & & & 0.75 \\
\hline Left & $1,951(50.44)$ & $105,747(50.69)$ & \\
\hline Right & $1,917(49.56)$ & $102,851(49.30)$ & \\
\hline Tumor Size $(\mathrm{cm})$ & & & 0.10 \\
\hline $0-2$ & $2,498(64.58)$ & $137,715(66.01)$ & \\
\hline $2-5$ & $1,161(30.02)$ & $59,339(28.44)$ & \\
\hline$>5$ & $209(5.40)$ & $11,567(5.54)$ & \\
\hline LN Status & & & 0.09 \\
\hline Negative & $2,523(65.23)$ & $139,399(66.82)$ & \\
\hline $1-3$ positive & $896(23.16)$ & $46,814(22.44)$ & \\
\hline 3 positive & $446(11.53)$ & $22,314(10.70)$ & \\
\hline AJCC Stage & & & 0.09 \\
\hline I & $1,910(49.37)$ & $106,580(51.08)$ & \\
\hline II & $1,439(37.20)$ & $75,584(36.23)$ & \\
\hline III & $519(13.42)$ & $26,457(12.68)$ & \\
\hline Histologic Grade & & & 0.05 \\
\hline I,II & $2,246(58.06)$ & $124,439(59.65)$ & \\
\hline III & $1,622(41.93)$ & $84,182(40.35)$ & \\
\hline ER Status & & & 0.33 \\
\hline Positive & $2,977(76.96)$ & $159,151(76.29)$ & \\
\hline Negative & $891(23.04)$ & $49,470(23.71)$ & \\
\hline PR Status & & & 0.11 \\
\hline Positive & $2,618(67.68)$ & $138,626(66.45)$ & \\
\hline
\end{tabular}




\begin{tabular}{llll}
\hline Negative & $1,250(32.32)$ & $69,995(33.55)$ & \\
Type of Surgery & & & $<\mathbf{0 . 0 1}$ \\
Lumpectomy & $1,946(50.31)$ & $122,191(58.57)$ & \\
Mastectomy & $1,893(48.94)$ & $84,292(40.40)$ & \\
None & $18(0.46)$ & $1,685(0.81)$ & \\
Radiation Therapy & & & $\mathbf{0 . 0 1}$ \\
Yes & $2,128(55.02)$ & $120,087(57.56)$ & \\
No & $1,740(44.98)$ & $88,534(42.44)$ & \\
\hline
\end{tabular}

Abbreviations: AJCC, American Joint Committee on Cancer; LN, lymph node; ER, estrogen receptor; PR, progesterone receptor.

${ }^{a}$ Bold type indicates statistical significance.

bot married includes never married, divorced, separated, and widowed.

to breast cancer risk factors similar to those of their Caucasian counterparts [5], indicating that data from Chinese populations cannot be extrapolated to Chinese immigrants living in the US. To our knowledge, there have been no studies specifically examining all of the available demographic, clinicopathologic, and outcomerelated breast cancer parameters in the population-based Surveillance, Epidemiology, and End Results (SEER) database for the Chinese-American cohort. Because Chinese Americans are the largest Asian subgroup in the US and $76 \%$ are immigrants [6], the SEER database could be used to compare the breast cancer survival rates of Caucasians and Chinese Americans living in the US to determine potential factors affecting these two races.

\section{RESULTS}

\section{Descriptive Statistics}

With a median follow-up time of 74 months, a total of 3868 Chinese and 208621 Caucasian women were identified in our analysis, according to the inclusion and exclusion criteria stated in METHODS. Among these patients, $378(9.77 \%)$ died from breast cancer and $531(13.73 \%)$ died from all causes among the Chinese individuals, whereas $21641(10.37 \%)$ patients died from breast cancer and 40698 (19.51\%) died from all causes among the Caucasians. All of the demographic and tumor characteristics are shown in Table 1.

Using the Chi-square test, we found significant differences in the demographic characteristics between the Caucasian and Chinese patients. The Chinese patients had a younger age at diagnosis, a later year of diagnosis, and a higher family income than the Caucasians. In addition, more Chinese patients remained married and resided in metropolitan areas. With regard to tumor characteristics, the Chinese patients seemed more inclined to receive mastectomy than lumpectomy compared with the Caucasians, and fewer Chinese patients underwent radiation therapy. The other tumor characteristics, including laterality, tumor size, lymph node (LN) status, American Joint Committee on Cancer (AJCC) stage, histologic grade, and estrogen receptor (ER) and progesterone receptor (PR) statuses, were similarly distributed between the two races.

\section{Comparison of Survival between Chinese and Caucasian Patients}

We first analyzed the unadjusted breast cancerspecific survival (BCSS) and overall survival (OS) rates of the Caucasian and Chinese patients using the Kaplan-
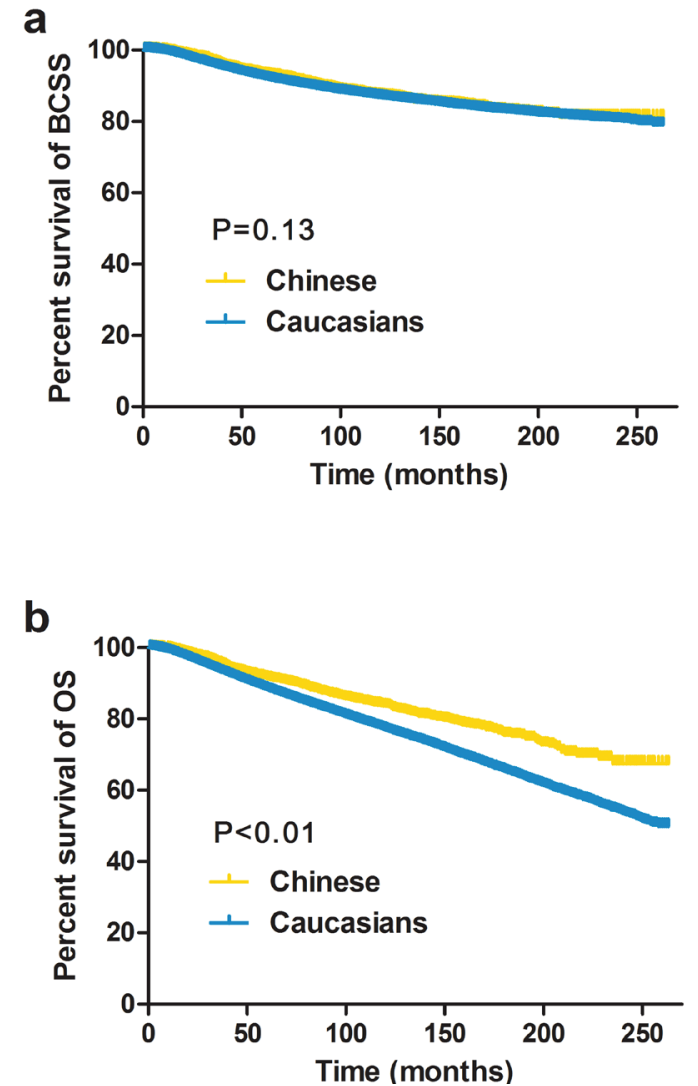

Figure 1: Kaplan-Meier estimates of breast cancerspecific survival (BCSS, a) and overall survival (OS, b) between Caucasian and Chinese female breast cancer patients residing in the US. 
Table 2: Univariate and multivariate Cox analysis of breast cancer-specific survival.

\begin{tabular}{|c|c|c|c|c|}
\hline \multirow{2}{*}{ Variables } & \multicolumn{2}{|c|}{ Univariate } & \multicolumn{2}{|c|}{ Multivariate $^{\mathrm{a}}$} \\
\hline & HR $(95 \% \mathrm{CI})$ & $\mathrm{P}^{\mathrm{b}}$ & HR $(95 \% \mathrm{CI})$ & $\mathrm{P}^{\mathrm{b}}$ \\
\hline Race & & 0.13 & & 0.07 \\
\hline Caucasian & 1 & & 1 & \\
\hline Chinese & $0.92(0.83-1.02)$ & & $0.90(0.81-1.01)$ & \\
\hline Age at Diagnosis (years) & & $<0.01$ & & $<0.01$ \\
\hline $20-39$ & 1 & & 1 & \\
\hline $40-59$ & $0.59(0.56-0.61)$ & & $0.85(0.82-0.89)$ & \\
\hline $60-79$ & $0.59(0.56-0.61)$ & & $1.10(1.05-1.15)$ & \\
\hline Year of Diagnosis & & $<0.01$ & & $<0.01$ \\
\hline 1990-1999 & 1 & & 1 & \\
\hline 2000-2009 & $0.65(0.63-0.66)$ & & $0.66(0.64-0.68)$ & \\
\hline Marital Status & & $<0.01$ & & $<0.01$ \\
\hline Married & 1 & & 1 & \\
\hline Not married ${ }^{\mathrm{c}}$ & $1.21(1.18-1.24)$ & & $1.18(1.15-1.21)$ & \\
\hline Family Income (\$) & & $<0.01$ & & $<0.01$ \\
\hline$<61,790$ & 1 & & 1 & \\
\hline $61,790-69,700$ & $0.99(0.96-1.03)$ & 0.70 & $0.94(0.91-0.98)$ & \\
\hline $69,700-85,140$ & $0.87(0.83-0.90)$ & & $0.91(0.87-0.95)$ & \\
\hline$\geq 85,140$ & $0.81(0.78-0.85)$ & & $0.87(0.83-0.90)$ & \\
\hline County Type & & $<0.01$ & & 0.04 \\
\hline Metropolitan & 1 & & 1 & \\
\hline Nonmetropolitan & $1.14(1.10-1.19)$ & & $1.05(1.00-1.10)$ & \\
\hline Laterality & & 0.01 & & 0.02 \\
\hline Left & 1 & & 1 & \\
\hline Right & $0.96(0.93-0.98)$ & & $0.97(0.94-1.00)$ & \\
\hline Tumor Size $(\mathrm{cm})$ & & $<0.01$ & & $<0.01$ \\
\hline $0-2$ & 1 & & 1 & \\
\hline $2-5$ & $3.61(3.50-3.72)$ & & $1.65(1.59-1.72)$ & \\
\hline$>5$ & $8.33(8.01-8.66)$ & & $2.35(2.21-2.48)$ & \\
\hline LN Status & & $<0.01$ & & $<0.01$ \\
\hline Negative & 1 & & 1 & \\
\hline 1-3 positive & $2.37(2.30-2.45)$ & & $1.50(1.44-1.57)$ & \\
\hline 3 positive & $6.60(6.40-6.81)$ & & $2.27(2.12-2.42)$ & \\
\hline Histologic Grade & & $<0.01$ & & $<0.01$ \\
\hline I,II & 1 & & 1 & \\
\hline III & $3.19(3.10-3.28)$ & & $1.70(1.65-1.76)$ & \\
\hline ER Status & & $<0.01$ & & $<0.01$ \\
\hline Positive & 1 & & 1 & \\
\hline Negative & $2.58(2.51-2.65)$ & & $1.40(1.35-1.45)$ & \\
\hline PR Status & & $<0.01$ & & $<0.01$ \\
\hline Positive & 1 & & 1 & \\
\hline Negative & $2.37(2.31-2.44)$ & & $1.38(1.33-1.43)$ & \\
\hline Type of Surgery & & $<0.01$ & & $<0.01$ \\
\hline Lumpectomy & 1 & & 1 & \\
\hline Mastectomy & $2.38(2.32-2.45)$ & & $1.12(1.08-1.16)$ & \\
\hline None & $6.86(6.24-7.55)$ & & $3.47(3.14-3.83)$ & \\
\hline Radiation Therapy & & $<0.01$ & & $<0.01$ \\
\hline Yes & 1 & & 1 & \\
\hline No & $1.37(1.33-1.40)$ & & $1.17(1.13-1.21)$ & \\
\hline
\end{tabular}

Abbreviations: AJCC, American Joint Committee on Cancer; ER, estrogen receptor; LN, lymph node; PR, progesterone receptor.

${ }^{a}$ Adjusted by Cox proportional hazard regression model including all factors, as categorized in Table 2 .

${ }^{b}$ Bold type indicates statistical significance.

${ }^{\mathrm{c}}$ Not married includes never married, divorced, separated, and widowed. 
Table 3: Univariate and multivariate Cox analyses of overall survival.

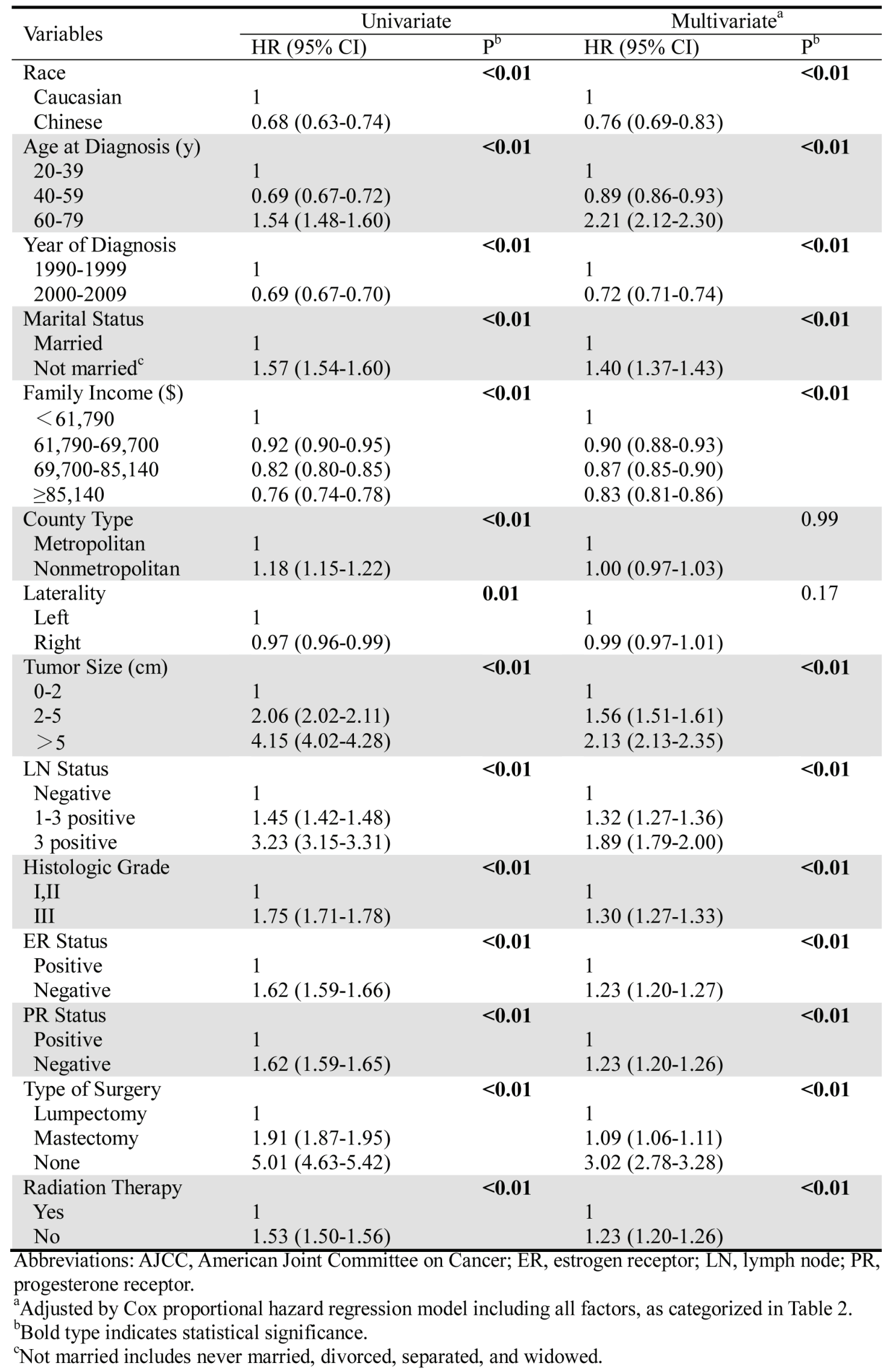


Table 4: Multivariate Cox proportional hazard regression model of breast cancer-specific

survival and overall-survival comparing Chinese with Caucasians stratified by

clinicopathologic variables.

\begin{tabular}{|c|c|c|c|c|}
\hline \multirow{3}{*}{ Variables $^{\mathrm{b}}$} & \multicolumn{4}{|c|}{ 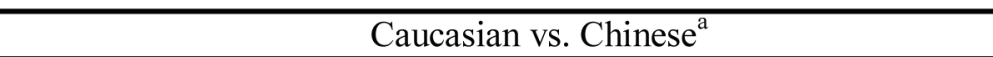 } \\
\hline & \multicolumn{2}{|c|}{ BCSS } & \multicolumn{2}{|c|}{ OS } \\
\hline & $\mathrm{HR}(95 \% \mathrm{CI})$ & $\mathrm{P}^{\mathrm{c}}$ & $\mathrm{HR}(95 \% \mathrm{CI})$ & $\mathrm{P}^{\mathrm{c}}$ \\
\hline \multicolumn{5}{|c|}{ Age at Diagnosis (y) } \\
\hline 20-39 & $0.83(0.64-1.08)$ & 0.16 & $0.81(0.62-1.04)$ & 0.10 \\
\hline $40-59$ & $1.03(0.89-1.18)$ & 0.72 & $0.93(0.82-1.06)$ & 0.26 \\
\hline $60-79$ & $0.73(0.59-0.91)$ & $<0.01$ & $0.61(0.52-0.70)$ & $<0.01$ \\
\hline \multicolumn{5}{|c|}{ Tumor Size $(\mathrm{cm})$} \\
\hline $0-2$ & $0.77(0.63-0.93)$ & $<0.01$ & $0.64(0.55-0.74)$ & $<0.01$ \\
\hline $2-5$ & $0.94(0.81-1.10)$ & 0.44 & $0.83(0.72-0.94)$ & $<0.01$ \\
\hline$>5$ & $0.94(0.73-1.21)$ & 0.63 & $1.06(0.83-1.36)$ & 0.63 \\
\hline \multicolumn{5}{|l|}{ LN Status } \\
\hline Negative & $0.85(0.71-1.02)$ & 0.09 & $0.66(0.57-0.76)$ & $<0.01$ \\
\hline $1-3$ positive & $1.00(0.83-1.21)$ & 0.99 & $0.83(0.70-0.98)$ & 0.03 \\
\hline 3 positive & $0.89(0.74-1.06)$ & 0.19 & $0.87(0.73-1.02)$ & 0.09 \\
\hline \multicolumn{5}{|c|}{ Histologic Grade } \\
\hline $\mathrm{I}, \mathrm{II}$ & $0.83(0.68-1.01)$ & 0.07 & $0.64(0.55-0.73)$ & $<0.01$ \\
\hline III & $0.93(0.82-1.06)$ & 0.28 & $0.85(0.76-0.95)$ & 0.01 \\
\hline \multicolumn{5}{|l|}{ ER Status } \\
\hline Positive & $0.98(0.85-1.12)$ & 0.71 & $0.77(0.68-0.86)$ & $<0.01$ \\
\hline Negative & $0.81(0.69-0.96)$ & 0.02 & $0.76(0.65-0.89)$ & $<0.01$ \\
\hline \multicolumn{5}{|l|}{ PR Status } \\
\hline Positive & $0.99(0.85-1.14)$ & 0.84 & $0.76(0.67-0.86)$ & $<0.01$ \\
\hline Negative & $0.83(0.71-0.96)$ & 0.01 & $0.76(0.66-0.86)$ & $<0.01$ \\
\hline \multicolumn{5}{|l|}{ Laterality } \\
\hline Left & $0.91(0.78-1.05)$ & 0.20 & $0.76(0.67-0.87)$ & $<0.01$ \\
\hline Right & $0.90(0.77-1.05)$ & 0.18 & $0.75(0.66-0.86)$ & $<0.01$ \\
\hline \multicolumn{5}{|c|}{ Type of Surgery } \\
\hline Lumpectomy & $0.98(0.81-1.18)$ & 0.79 & $0.77(0.66-0.91)$ & $<0.01$ \\
\hline Mastectomy & $0.88(0.77-1.00)$ & 0.06 & $0.75(0.67-0.84)$ & $<0.01$ \\
\hline None & $1.28(0.57-2.88)$ & 0.56 & $1.22(0.58-2.59)$ & 0.60 \\
\hline \multicolumn{5}{|c|}{ Radiation Therapy } \\
\hline Yes & $0.95(0.82-1.10)$ & 0.49 & $0.82(0.73-0.94)$ & $<0.01$ \\
\hline No & $0.86(0.73-1.00)$ & 0.06 & $0.70(0.61-0.80)$ & $<0.01$ \\
\hline
\end{tabular}

Abbreviations: ER, estrogen receptor; LN, lymph node; PR, progesterone receptor.

${ }^{\mathrm{a}}$ Using Caucasian as a reference.

${ }^{\mathrm{b}}$ Adjusted by Cox proportional hazard regression model including all factors, as categorized in Table 2 .

${ }^{c}$ Bold type indicates statistical significance.

Meier method and log-rank test (Figure 1). Compared to the Caucasians, the Chinese patients had significantly better OS rates $(\mathrm{P}<0.01)$ but similar BCSS rates $(\mathrm{P}=0.13)$.

The results of the BCSS and OS analyses via univariate and multivariate Cox proportional hazard regression models are shown in Table 2 and Table 3, respectively. The BCSS was not significantly different between the two races (univariate $\mathrm{P}=0.13$, multivariate $\mathrm{P}=0.10$ ). In contrast, all of the other characteristics, including older age, a later year of diagnosis, continued married status, a higher family income, living in a metropolitan area, a tumor on the right side, a tumor size of 0-2 cm, a negative LN status, higher differentiation, ER and PR positivity, increased acceptance of lumpectomy and receipt of radiation therapy, were independently associated with increased BCSS (Table 2), according to univariate and multivariate analyses. These factors were also related to increased OS (Table 3), and the Chinese patients showed significantly better OS than the Caucasians (using the Caucasians as a reference, 
univariate: hazard ratio, $\mathrm{HR}=0.68,95 \%$ confidence interval, CI: 0.63-0.74, $\mathrm{P}<0.01$; multivariate: $\mathrm{HR}=0.76$, 95\% CI: 0.69-0.83, $\mathrm{P}<0.01)$.

\section{Comparison of Survival by Stratification Analysis}

We considered that there might be confounding factors affecting breast cancer outcomes between the Caucasian and Chinese patients. Therefore, we further performed multivariate analysis, stratifying according to the potential characteristics (Table 4). Although there was no difference between the two races with regard to BCSS, patients between the ages of 60 and 79 years old (using Caucasian as reference: $\mathrm{HR}=0.73,95 \% \mathrm{CI}: 0.59-0.91, \mathrm{P}<$ $0.01)$, those with a tumor size of $0-2 \mathrm{~cm}(\mathrm{HR}=0.77,95 \%$ CI: $0.63-0.93, \mathrm{P}<0.01$ ), and those who were ER-negative ( $\mathrm{HR}=0.81,95 \% \mathrm{CI}: 0.69-0.96, \mathrm{P}=0.02)$ and $\mathrm{PR}$-negative ( $\mathrm{HR}=0.83,95 \%$ CI: 0.71-0.96, $\mathrm{P}=0.01)$ showed significant differences in BCSS, suggesting that the Chinese patients in these subgroups had better survival than the Caucasians. In contrast, most of the stratified subgroups showed significant differences in OS, except for the patients aged 60-79 years old and those with a tumor size of $>5 \mathrm{~cm}, 3$ positive LNs, and no history of surgery $(\mathrm{P}>0.05)$. The Chinese patients in these groups did not exhibit superior OS rates to their Caucasian counterparts.

\section{DISCUSSION}

In our evaluation of more than 200000 primary breast cancer patients reported to population-based cancer registries across the US by the SEER program, we found that the BCSS of Chinese patients living in the US was similar to that of Caucasians. Previous studies that have mainly focused on all Asians living in the US, investigating disparities among unique Asian subgroups, have reported similar results to those of the present study [4, 7], and our findings contributed to these previous studies by providing a comprehensive assessment of differences in breast cancer outcomes between Caucasian and Chinese women residing in the US. Moreover, we found that the Chinese patients had distinctively better OS than the Caucasians, even when stratified by traditional clinicopathologic characteristics, including age, year of diagnosis, marital status, family income, type of residential county, tumor size, LN status, ER and PR statuses, histological grade, surgical treatment and radiation therapy.

Chinese immigrants are considered to have worse socioeconomic status (SES) than their Caucasian counterparts [8], suggesting that they might have limited access to medical insurance and appropriate treatments. In addition, they appear less likely to have their cancerrelated issues resolved due to their traditional cultural beliefs $[9,10]$. Interestingly, the Chinese patients in our study had much higher family incomes than the
Caucasians, and more of them resided in metropolitan counties. Furthermore, they received breast-conserving surgery (BCS) $(50.31 \%$, Table 2$)$ much more frequently than the Chinese natives did (12.2\%, [11]). Additionally, the rates of BCS and radiation therapy for the Chinese patients residing in the US were similar to those of their Caucasian counterparts (BCS: 50.31 vs. 58.57 ; radiation therapy: 55.02 vs. 57.56 ), although the differences were significant. Considering these findings, we inferred that Chinese patients living in the US, or at least those who were registered in the SEER database, represented a welleducated, higher SES group of Chinese individuals who were able to obtain optimal medical resources similar to those obtained by Caucasians. Despite discrepancies in the demographic characteristics, there were no significant differences in tumor characteristics, including tumor size, LN status, ER and PR statuses, histological grade, and AJCC stage. Considering the differences in tumor biology between these two ethnicities, we suggest that the worse survival rate of Chinese patients living in China can mainly be attributed to worse SES and not tumor behavior itself. Chinese patients may experience improved outcomes with more advanced treatment. This notion is supported by the prediction that the future survival curve in China will be similar to that in Western countries with the development of the Chinese economy [1].

In our investigation, we found that the Chinese patients had a higher OS rate than the Caucasians, consistent with previous findings obtained from a study of Asian women aged 18-39 years old, which showed that Chinese Americans have better 5-year and 10-year OS rates than Caucasians do [12]. After stratifying by potentially confounding factors, this advantage still existed, except for those patients aged 60-79 old and those with a tumor size of $>5 \mathrm{~cm}, 3$ positive LNs, and no history of surgery. The superior OS of the Chinese patients was markedly attenuated in these groups, which might have been due to their more advanced and aggressive breast cancers, resulting in poor prognoses that neutralized the increase in OS.

Because the BCSS rates were similar between the two races assessed in our study, the difference in OS was not caused by breast cancer-related deaths. Notably, the overall age distribution of the Chinese women was less than that of the Caucasians (Table 2), which might have been partially due to immigration patterns because the Chinese population experienced a rapid increase in younger-aged individuals in the latter half of the twentieth century [12]. Additionally, this finding reflects that Chinese women are typically younger at the time of breast cancer diagnosis [11]. Due to their younger ages, Chinese women might have fewer complications, such as heart disease, cerebrovascular disease, and chronic lower respiratory disease, resulting in greater OS. However, in our further stratification analysis, the patients aged 2039 and 40-59 years old did not exhibit any differences in 
survival, whereas the Chinese patients aged 60-79 years old had better survival than the Caucasians did, in terms of both BCSS and OS. A possible explanation for this finding was that older Chinese patients might be more inclined to adopt a traditional Chinese lifestyle, resulting in reduced fat intake and increased physical activity. With healthy life habits, older Chinese patients achieve a lower body mass index and a reduction in obesity, both of which are associated with improved breast cancer survival $[13,14]$.

Our study had the advantage of including a sizable number of breast cancer patients reported to cancer registries, with near completeness in terms of registration and follow-up data [15]. These large numbers allowed us to examine racial differences in survival among subgroups of patients with different demographic and tumor characteristics. There are clearly important limitations inherent in using a large population-based dataset. Data pertaining to SES, family history of breast cancer, lifestyle factors, human epidermal growth factor receptor 2 status, medical insurance, and the administration of neoadjuvant or adjuvant systemic therapy were limited, preventing us from evaluating these factors as potential confounders or effect modifiers of the relationships observed. The long duration of our study (1990-2009) might have affected the results due to missing data or changes in management. However, neither of these factors would be expected to result in a sufficiently strong bias to override the known impacts of tumor size, LN status, age at diagnosis, race, and SES, all of which were included in the multivariate model.

In conclusion, our study demonstrated that the presenting demographic features of breast cancer patients differed between Caucasian and Chinese women residing in the US. However, the tumor characteristics in the two groups were similar, suggesting that Chinese patients will have similar outcomes once they attain better medical resources. Regardless of these factors, the Chinese women had better OS than their Caucasian counterparts. Further pre-clinical and clinical studies should be conducted to confirm these conclusions and to clarify the underlying mechanisms.

\section{MATERIALS AND METHODS}

\section{Ethical statement}

This study was approved by the Ethical Committee of the Shanghai Cancer Center of Fudan University. The data released from the SEER database did not require informed patient consent because cancer is a reportable disease in every state in the US.

\section{Patient selection}

The data for this study were derived from the SEER program of the US National Cancer Institute (SEER 18 Regs Research Data and Hurricane Katrina Impacted Louisiana Cases, Nov 2013 Sub [1973-2011 varying]). We selected female patients diagnosed with primary invasive breast cancer from January 1, 1990, through December 31, 2009. Race/ethnicity was our primary exposure of interest, and we selected Caucasian and Chinese individuals residing in the US according to the SEER algorithm [16]. Women with other combinations of race/ethnicity were excluded.

The specific inclusion criteria were as follows: female sex, Chinese or Caucasian race/ethnicity, age of diagnosis between 20 and 79 years old, diagnosis between 1990 and 2009, breast cancer as the primary and only cancer diagnosis, unilateral breast cancer, pathologically confirmed infiltrating ductal carcinoma (IDC, ICD-O-3 $8500 / 3)$, AJCC stages I to III, histological grades I to III, and known tumor size, as well as LN status, ER and PR statuses. Women who were diagnosed with breast cancer at death or by autopsy only and those with other first primary cancers, in situ disease, histological grade IV (SEER program code: undifferentiated or anaplastic), and no record of surgery type or radiation therapy were excluded from this analysis. Patients diagnosed with breast cancer before 1990 were not included because the SEER database did not record data on ER and PR statuses until 1990. Additionally, patients diagnosed with breast cancer after 2010 were not included because the database was only updated through December 31, 2010, and we wanted to ensure an adequate follow-up duration.

\section{Statistical analysis}

The covariates included in our analyses were limited to those available in the SEER program data. Demographic statistics included race, age at diagnosis, year of diagnosis, marital status, family income, and county metropolitan status. Tumor characteristics included laterality, tumor size, lymph node status, AJCC stage, histological grade, ER status, PR status, surgery type and radiotherapy. The primary outcomes of our study were BCSS and OS. BCSS was defined as the time from the date of diagnosis to the date of death due to breast cancer or the last follow-up, and OS was measured from the date of diagnosis to the date of death due to all causes (including breast cancer) or the last follow-up.

Patient and tumor characteristics between the different races were compared using Pearson's Chi-square test. Survival curves were generated using the KaplanMeier method, and the log-rank test was performed to compare the unadjusted BCSS and OS rates between the two races. Adjusted HRs with 95\% CIs were estimated 
using Cox proportional hazard regression models. P-values of $<0.05$ were considered significant. SPSS software, version 18.0 (SPSS, Inc., Chicago, IL, US), was used for all of the analyses.

\section{ACKNOWLEDGMENTS}

This work was supported by grants from the Provincial Natural Science Foundation of Fujian, China (2012J01357); the Research Project of Fudan University Shanghai Cancer Center (YJ201401), the National Natural Science Foundation of China (81372848, 81370075); the Municipal Project for Developing Emerging and Frontier Technology in Shanghai Hospitals (SHDC12010116); the Cooperation Project of Conquering Major Diseases in Shanghai Municipality Health System (2013ZYJB0302); the Innovation Team of Ministry of Education (IRT1223); and the Shanghai Key Laboratory of Breast Cancer (12DZ2260100).

\section{COMPETING INTERESTS}

The authors have declared that no competing interests exist.

\section{Editorial note}

This paper has been accepted based in part on peerreview conducted by another journal and the authors' response and revisions as well as expedited peer-review in Oncotarget.

\section{REFERENCES}

1. Fan L, Strasser-Weippl K, Li J-J, St Louis J, Finkelstein DM, Yu K-D, Chen W-Q, Shao Z-M and Goss PE. Breast cancer in China. Lancet Oncol. 2014; 15:e279-e289.

2. Jemal A, Siegel R, Xu J and Ward E. Cancer statistics, 2010. CA Cancer J Clin. 2010; 60:277-300.

3. Fan L, Zheng Y, Yu K-D, Liu G-Y, Wu J, Lu J-S, Shen K-W, Shen Z-Z and Shao Z-M. Breast cancer in a transitional society over 18 years: trends and present status in Shanghai, China. Breast Cancer Res Treat. 2009; 117:409-416.

4. Pineda MD, White E, Kristal AR and Taylor V. Asian breast cancer survival in the US: a comparison between Asian immigrants, US-born Asian Americans and Caucasians. Int J Epidemiol. 2001; 30:976-982.

5. Moran MS, Gonsalves L, Goss DM and Ma S. Breast cancers in U.S. residing Indian-Pakistani versus nonHispanic White women: comparative analysis of clinicalpathologic features, treatment, and survival. Breast Cancer Res Treat. 2011; 128:543-551.

6. Elizabeth M. Hoeffel, Sonya Rastogi, Myoung Ouk Kim and Hasan Shahid. The Asian Population: 2010. US Census Bureau; 2012.

7. Min Yi, Peijun Liu, Xu Li, Elizabeth A. Mittendorf and He J. Comparative Analysis of Clinicopathologic Features, Treatment, and Survival of Asian Women With a Breast Cancer Diagnosis Residing in the United States. Cancer. 2012; 118:4117-4125.

8. Judy Huei-yu Wang, Inez F. Adams, Reginald TuckerSeeley, Scarlett Lin Gomez, Laura Allen and Huang E. A mixed method exploration of survivorship among Chinese American and non-Hispanic White breast cancer survivors: the role of socioeconomic well-being. Qual Life Res. 2013; 22:2709-2720.

9. Judy Huei-yu Wang, Inez Adams, Ellen Huang, Kimlin Ashing-Giwa, Scarlett Lin Gomez and Allen L. Physical distress and cancer care experiences among ChineseAmerican and non-Hispanic White breast cancer survivors. Gynecol Oncol. 2012; 124:383-388.

10. Ashing-Giwa KT, Padilla G, Tejero J, Kraemer J, Wright K, Coscarelli A, Clayton S, Williams I and Hills D. Understanding the breast cancer experience of women: A qualitative study of African American, Asian American, Latina and Caucasian cancer survivors. Psycho-Oncology. 2004; 13:408-428.

11. Li J, Zhang BN, Fan JH, Pang Y, Zhang P, Wang SL, Zheng S, Zhang B, Yang HJ, Xie XM, Tang ZH, Li H, Li JY, He JJ and Qiao YL. A nation-wide multicenter 10-year (19992008) retrospective clinical epidemiological study of female breast cancer in China. BMC Cancer. 2011; 11:364.

12. Gathani T, Ali R, Balkwill A, Green J, Reeves G, Beral $\mathrm{V}$ and Moser KA. Ethnic differences in breast cancer incidence in England are due to differences in known risk factors for the disease: prospective study. $\mathrm{Br} \mathrm{J}$ Cancer. 2013; 110:224-229.

13. Patterson RE, Cadmus LA, Emond JA and Pierce JP. Physical activity, diet, adiposity and female breast cancer prognosis: a review of the epidemiologic literature. Maturitas. 2010; 66:5-15.

14. Thiebaut AC, Kipnis V, Chang SC, Subar AF, Thompson FE, Rosenberg PS, Hollenbeck AR, Leitzmann M and Schatzkin A. Dietary fat and postmenopausal invasive breast cancer in the National Institutes of Health-AARP Diet and Health Study cohort. J Natl Cancer Inst. 2007; 99:451-462.

15. Wong-Ho Chow, Brian Shuch, W. Marston Linehan and Susan S. Devesa. Racial Disparity in Renal Cell Carcinoma Patient Survival According to Demographic and Clinical Characteristics. Cancer. 2012; 119:3100.

16. Bach PB, Guadagnoli E, Schrag D, Schussler N and Warren JL. Patient demographic and socioeconomic characteristics in the SEER-Medicare database applications and limitations. Med Care. 2002; 40:IV-19-25. 